\title{
Probing neutrino mass with multilepton production at the Tevatron
}

\author{
Oscar Éboli*† \\ Instituto de Física, Universidade de São Paulo, São Paulo - SP, Brazil. \\ E-mail: eboli@fma.if.usp.br
}

\begin{abstract}
We analyse the production of multileptons in the simplest supergravity model with bilinear violation of $R$ parity at the Fermilab Tevatron. We show that the presence of bilinear $R$-parity violating $(\mathrm{BRpV})$ interactions enhances the supersymmetric multilepton signals over most of the parameter space, specially at moderate and large $m_{0}$, despite the small $R$-parity violating couplings needed to generate the neutrino masses indicated by current atmospheric neutrino data.
\end{abstract}

\section{Introduction}

It has been suggested long ago that neutrino masses and supersymmetry may be deeply tied together [1]. Indeed, SUSY models exhibiting $R$-parity violation can lead to neutrino masses and mixings 22 in agreement with the current solar and atmospheric neutrino data. A very interesting property of neutrino mass models based on $R$-parity violation is that they can be tested at colliders [3, 目, 5]. In this work, we study the production of multileptons ( $\geq 3 \ell$ with $\ell=e$ or $\mu$ ) at the Fermilab Tevatron within the framework of the simplest supergravity (SUGRA) model without $R$-parity conservation [6.

At the Fermilab Tevatron the production of charginos and neutralinos provides the largest reach in SUGRA models with universal soft breaking terms at unification since the masses of the lighter chargino $\left(\tilde{\chi}_{1}^{ \pm}\right)$and the lighter neutralinos $\left(\tilde{\chi}_{1}^{0}\right.$ and $\left.\tilde{\chi}_{2}^{0}\right)$ are considerably smaller than the gluino and squark ones over a large range of the parameter space [7. In $R$-parity conserving scenarios, a promising signal for SUGRA at the Tevatron is the production of $\tilde{\chi}_{2}^{0} \tilde{\chi}_{1}^{ \pm}$pairs and their subsequent decay into three charged leptons in association with missing energy due to the undetected lightest supersymmetric particle (LSP), which turns out to be $\tilde{\chi}_{1}^{0}$. In the presence of $R$-parity violation, the LSP is no longer stable,

\footnotetext{
${ }^{*}$ Speaker.

${ }^{\dagger}$ This work has been done in collaboration with M. B. Magro, F. de Campos, W. Porod, D. Restrepo, and J. W. F. Valle; for further information see M. Magro et al., JHEP 0309, 071 (2003).
} 
giving rise to events usually containing more visible particles. Our goal in this work is to determine the impact of $R$-parity violation on SUSY multilepton signals, for example in the production of three or more electrons or muons.

Here we consider a SUGRA model that includes the following bilinear terms in the superpotential [6]

$$
W_{B R p V}=W_{M S S M}-\varepsilon_{a b} \epsilon_{i} \widehat{L}_{i}^{a} \widehat{H}_{u}^{b},
$$

where the last term violates $R$ parity. In order to reproduce the values of neutrino masses indicated by current neutrino data we must have $\left|\epsilon_{i}\right| \ll|\mu|$, where $\mu$ denotes the SUSY bilinear mass parameter [2]. The relevant bilinear terms in the soft supersymmetry breaking sector are

$$
V_{\text {soft }}=m_{H_{u}}^{2} H_{u}^{a *} H_{u}^{a}+m_{H_{d}}^{2} H_{d}^{a *} H_{d}^{a}+M_{L_{i}}^{2} \widetilde{L}_{i}^{a *} \widetilde{L}_{i}^{a}-\varepsilon_{a b}\left(B \mu H_{d}^{a} H_{u}^{b}+B_{i} \epsilon_{i} \widetilde{L}_{i}^{a} H_{u}^{b}\right),
$$

where the terms proportional to $B_{i}$ are the ones that violate $R$ parity. The explicit $R$-parity violating terms induce vacuum expectation values (vev) $v_{i}, i=1,2,3$ for the sneutrinos, in addition to the two Higgs vev's $v_{u}$ and $v_{d}$. For the sake of simplicity, we assume the approximation where $R$ parity is violated only in the third generation. From the point of view of the analysis presented below, the breaking of $R$ parity only in the third generation corresponds to the worst-case-scenario: the breaking of $R$ parity in the muon channel would produce muons directly, not just as tau decay products, leading to an enhanced multilepton (multi-muon) signal.

The parameter space of our SUGRA model, which exhibits $R$-parity violation only in the third generation, is

$$
m_{0}, m_{1 / 2}, \tan \beta, \operatorname{sign}(\mu), A_{0}, \epsilon_{\tau}, \text { and } m_{\nu_{3}},
$$

where $m_{1 / 2}$ and $m_{0}$ characterise the common gaugino mass and scalar soft SUSY breaking masses at the unification scale, $A_{0}$ is the common trilinear term, and $\tan \beta$ is the ratio between the Higgs field vev's. It is convenient to characterise the BRpV sector by the bilinear superpotential term $\epsilon_{\tau}$ and the neutrino mass $m_{\nu_{3}}$.

The search for supersymmetry at the Tevatron is changed with respect to the $R$-parity conserving case since the presence of $\mathrm{BRpV}$ induces a mixing between the neutrinos and neutralinos, giving rise to $R$-parity violating decays of the LSP. In our model, the lightest neutralino presents leptonic decays $\tilde{\chi}_{1}^{0} \rightarrow \nu \ell^{+} \ell^{\prime-}$, semi-leptonic ones $\tilde{\chi}_{1}^{0} \rightarrow \nu q \bar{q}$ or $\ell q \bar{q}$, and the invisible mode $\tilde{\chi}_{1}^{0} \rightarrow \nu \nu \nu$ [4]. The expected $\tilde{\chi}_{1}^{0}$ lifetime and decay lengths depend both on the magnitude of $R$-parity breaking parameters and the chosen values of the SUGRA parameters.

As an illustration, Fig. 1 s shows the lightest neutralino decay length as a function of its mass for $A_{0}=0, \mu>0, \tan \beta=3$, and $\epsilon_{\tau}=0.22 \mathrm{GeV}$. In this figure, the solid lines stand for the tree-level neutrino mass $m_{\nu_{3}}=0.05 \mathrm{eV}$, corresponding to the best fit atmospheric scale as given in Ref. [8], and we chose $m_{0}=200$ and $700 \mathrm{GeV}$. The bands in these figures were obtained by taking $m_{\nu_{3}}$ within the $3 \sigma$ allowed atmospheric mass splitting of Ref. 87 . From Fig. 1 we can see clearly that the LSP decay length is shorter for larger LSP masses, as expected. Furthermore, irrespective of the smallness of the neutrino mass indicated by 


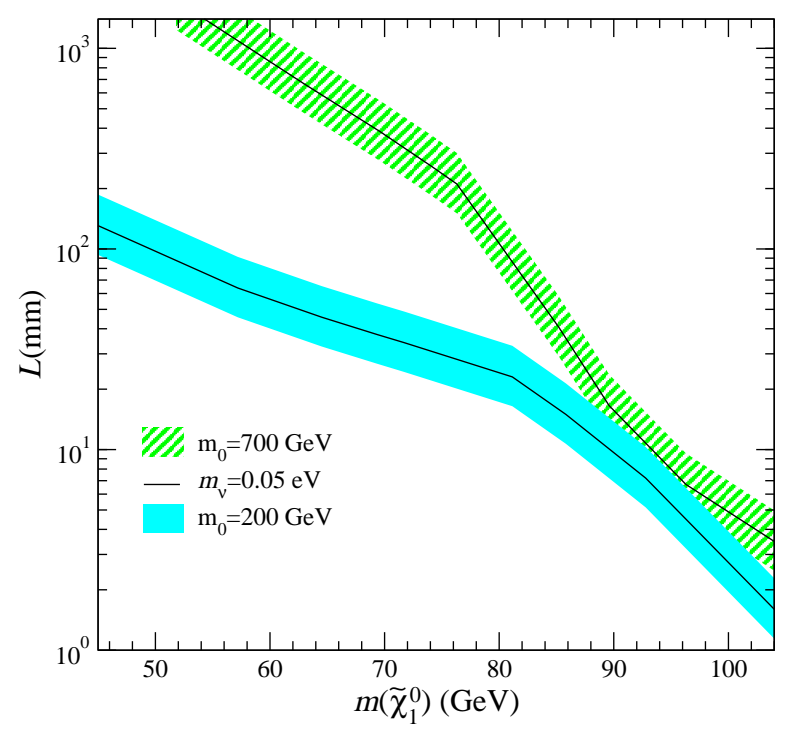

Figure 1: $\tilde{\chi}_{1}^{0}$ decay length versus LSP mass for $A_{0}=0, \mu>0$ and $\tan \beta=3$ for fixed BRpV parameters: $\epsilon_{\tau}=0.22 \mathrm{GeV}, m_{\nu_{3}}=0.05 \mathrm{eV}$ (solid lines) and current atmospheric $3 \sigma$ band (shaded bands)

the atmospheric oscillation data, the LSP $\left(\tilde{\chi}_{1}^{0}\right)$ decays inside the detector in a large portion of the parameter space, specially for small $m_{0}$. For further details on the neutralino decays see Ref. [9].

\section{Signal, Backgrounds, and Selection Cuts}

In $R$-parity conserving scenarios, trilepton production at the Tevatron proceeds via $p \bar{p} \rightarrow$ $\tilde{\chi}_{2}^{0} \tilde{\chi}_{1}^{ \pm}$with $\tilde{\chi}_{1}^{ \pm} \rightarrow \ell \nu \tilde{\chi}_{1}^{0}, \tilde{\chi}_{2}^{0} \rightarrow \ell^{+} \ell^{-} \tilde{\chi}_{1}^{0}$, and the LSP $\left(\tilde{\chi}_{1}^{0}\right)$ leaving the detector undetected. The main SM backgrounds for trilepton production are displayed in Table 1. In order to suppress these backgrounds, we have imposed the soft cuts SC2 defined in Refs. [7, 10]:

C1: We required the presence of three isolated leptons ( $e$ or $\mu$ ) with a hadronic $E_{T}$ smaller that $2 \mathrm{GeV}$ in a cone of size $\Delta R=0.4$ around the lepton [11];

C2: We required the most energetic lepton to satisfy $\left|\eta_{\ell_{1}}\right|<1.0$ and $p_{T}\left(\ell_{1}\right)>11 \mathrm{GeV}$;

C3: The second (third) most energetic lepton must satisfy $\left|\eta_{\ell_{2 / 3}}\right|<2.0$ and $p_{T}\left(\ell_{2(3)}\right)>7$ (5) $\mathrm{GeV}$; 
C4: We required the missing transverse energy to be larger than $25 \mathrm{GeV}$;

C5: We vetoed events exhibiting a $\ell^{+} \ell^{-}$pair with an invariant mass smaller than $20 \mathrm{GeV}$ and larger than $81 \mathrm{GeV}$ (this avoids both $\mathrm{Z}$ boson and QED contributions);

C6: We vetoed events with a transverse mass of a charged lepton and missing transverse energy between $60 \mathrm{GeV}$ and $85 \mathrm{GeV}$ in order to suppress $W$ decays.

\begin{tabular}{|l|c|}
\hline $\mathrm{BG}(\mathrm{fb})$ & $\sigma(\mathrm{fb})$ \\
\hline \hline $\mathrm{WZ}(\mathrm{Z} \rightarrow \tau \tau)$ & 0.17 \\
\hline $\mathrm{W}^{*} \mathrm{Z}^{*}, \mathrm{~W}^{*} \gamma \rightarrow l l \bar{l}$ & 0.12 \\
\hline $\mathrm{W}^{*} \mathrm{Z}^{*}, \mathrm{~W}^{*} \gamma \rightarrow l l^{\prime} l^{\prime}$ & 0.15 \\
\hline$t \bar{t}$ & 1.15 \\
\hline $\mathrm{Z}^{*} \mathrm{Z}^{*}$ & 0.05 \\
\hline total & 1.64 \\
\hline \hline
\end{tabular}

Table 1: Background cross sections in $\mathrm{fb}$ for the trilepton signal at the Tevatron Run II after kinematical cuts discussed in the text.
In our analysis, the signal and backgrounds were generated using PYTHIA [12], except for the $W Z^{\star}\left(\gamma^{\star}\right)$ which was computed using the complete matrix elements. The cross section for the SM backgrounds after cuts are shown in Table 1. We can see from this table that the most important background is the $t \bar{t}$ production, accounting for $70 \%$ of the total background.

In our BRpV model there are more SUSY reactions that can contribute to the trilepton signal than in the $R$-parity conserving case, since the $\tilde{\chi}_{1}^{0}$ decays can give rise to charged leptons. In the parameter range interesting for neutrino physics $R$-parity violating decay modes are only important for the LSP decays and can safely be neglected for the other SUSY particles. We have used the branching ratios generated by PYTHIA except for the the lightest and second neutralinos, which were evaluated separately and inputed into PYTHIA.

Assuming that gluinos and squarks are too heavy to be produced at the Tevatron, we considered the following processes:

$$
p \bar{p} \rightarrow \tilde{\ell} \tilde{\ell}^{\star} \quad, \quad \tilde{\nu} \tilde{\ell} \quad, \quad \tilde{\chi}_{i}^{0} \tilde{\chi}_{j}^{0}(i(j)=1,2) \quad, \quad \tilde{\chi}_{1}^{+} \tilde{\chi}_{1}^{-} \quad \text { and } \quad \tilde{\chi}_{i}^{0} \tilde{\chi}_{1}^{ \pm}(i=1,2) .
$$

The $\tilde{\chi}_{1}^{0}$ decays can contain charged leptons, and therefore, we should also analyse multilepton $(\geq 4 \ell)$ production. In order to extract this signal, we applied the cuts C1, C3, C5, and C2 but accepting leptons with $|\eta(\ell)|<3$. We also required the presence of an additional isolated lepton with $p_{T}>5 \mathrm{GeV}$ and demanded the missing transverse energy to be larger than $20 \mathrm{GeV}$. The main SM backgrounds for this process are $t \bar{t}, W Z$, and $Z Z$ productions whose cross sections after cuts are shown in Table 2 .

We can see from Fig. 1 that the lightest neutralino might not decay inside the detector depending on the point of the parameter space. If it decays inside the tracking system, it can give rise to spectacular events exhibiting displaced vertices without an incoming track associated to it. In our analyses, we did not look for displaced vertices since the corresponding backgrounds depend upon details of the detector. This possibly is a conservative hypothesis since this kind of events should present a small background, leading to a larger reach of the Tevatron. Nevertheless, we kept track of the position of the neutralino decay and accepted events where the neutralino decays inside the tracking system, rejecting all events where one of the neutralinos decays outside a cylinder around the beam pipe of 
radius $0.5 \mathrm{~m}$ and length $2.0 \mathrm{~m}$; we named this requirement $\mathrm{C} 7$. Again, this is another conservative estimate of the expected $\mathrm{BRpV}$ signal.

\begin{tabular}{|l|c|}
\hline BG $(\mathrm{fb})$ & $\sigma(\mathrm{fb})$ \\
\hline \hline $\mathrm{WZ}$ & 0.01 \\
\hline $\mathrm{Z}^{*} \mathrm{Z}^{*}$ & 0.10 \\
\hline$t \bar{t}$ & 0.16 \\
\hline total & 0.27 \\
\hline \hline
\end{tabular}

Table 2: Background cross sections in $\mathrm{fb}$ for the multilepton signal at the Fermilab Tevatron Run II after kinematical cuts discussed in the text.

The trilepton cross section is always dominated by the $\tilde{\chi}_{1}^{+} \tilde{\chi}_{1}^{-}$and $\tilde{\chi}_{2}^{0} \tilde{\chi}_{1}^{ \pm}$productions, with the second process being about $20 \%$ to $50 \%$ larger than the first. For example, these reactions are responsible for approximately $99 \%$ of the cross section at large $m_{0}$. Note that the first process can contribute to the trilepton signal only in $R$-parity violating scenarios. Moreover, at small and moderate $m_{0}(\lesssim 400 \mathrm{GeV})$, the $\tilde{\chi}_{1}^{0} \tilde{\chi}_{1}^{ \pm}$production is responsible for approximately $5-10 \%$ of the signal cross section and the production of sleptons also gives a sizable contribution.

Here we present the best scenario case where $\tan \beta$ and $\epsilon_{\tau}$ are small; for a more complete discussion see Ref. [9]. In Fig. 2, we present the region of the $m_{0} \otimes m_{1 / 2}$ plane that can be probed at the Tevatron for $\tan \beta=3$ and $\mathrm{BRpV}$ parameters $\epsilon_{\tau}=7 \times 10^{-4} \mathrm{GeV}$ and $m_{\nu_{3}}=0.05 \mathrm{eV}$. For these values of the parameters, the signal cross section is dominated by $\tilde{\chi}_{1}^{+} \tilde{\chi}_{2}^{0}$ production followed by $\tilde{\chi}_{1}^{+} \rightarrow \tilde{\chi}_{1}^{0} l^{+} \nu, \tilde{\chi}_{2}^{0} \rightarrow \tilde{\chi}_{1}^{0} q q$ and the LSP $\tilde{\chi}_{1}^{0}$ decays mainly into $\tau u \bar{d}$. As expected, the long lifetime of the neutralino reduces considerably the signal in the shaded area of Fig. 2 after we apply the cut C7. Therefore, we might be able to further probe this region by looking for displaced vertices, and consequently enhance the Tevatron reach.

It is interesting to compare our results presented in Fig. 2 with the ones in Ref. [7]. First of all, the presence of $\mathrm{BRpV}$ interactions reduces the Tevatron reach in the trilepton channel for small values of $m_{0}$. This happens because there are some competing effects in this region of parameters: on the one hand there are new contributions to the trilepton process due to LSP decay and on the other hand, the decay of the neutralinos produce a larger hadronic activity, worsening the lepton isolation, and reducing the missing $E_{T}$ compared with the MSSM case. Besides that, the leptons from the $\tilde{\chi}_{1}^{0}$ decay can give rise to additional isolated leptons which can contribute to the trilepton signal or, alternatively, can suppress it due to the presence of more than three isolated leptons. The last effect and the larger hadronic activity reduce the trilepton signal at small $m_{0}$ in the BRpV model. In contrast, as can be seen from Fig. 2, the trilepton reach at large $m_{0}$ always tends to increase with respect to the MSSM expectation. This follows from the fact that the 


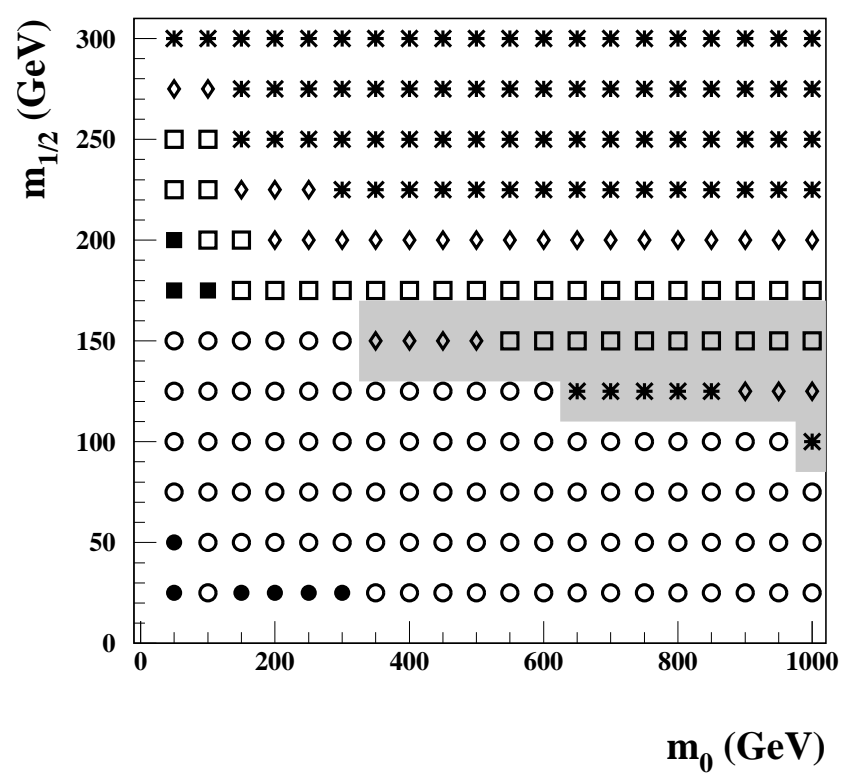

Figure 2: Reach of Fermilab Tevatron Run II using the trilepton signal in the $m_{0} \otimes m_{1 / 2}$ plane for $A_{0}=0, \tan \beta=3, \mu>0, \epsilon_{\tau}=7 \times 10^{-4} \mathrm{GeV}$, and $m_{\nu_{3}}=0.05 \mathrm{eV}$. The conventions are described in the text.
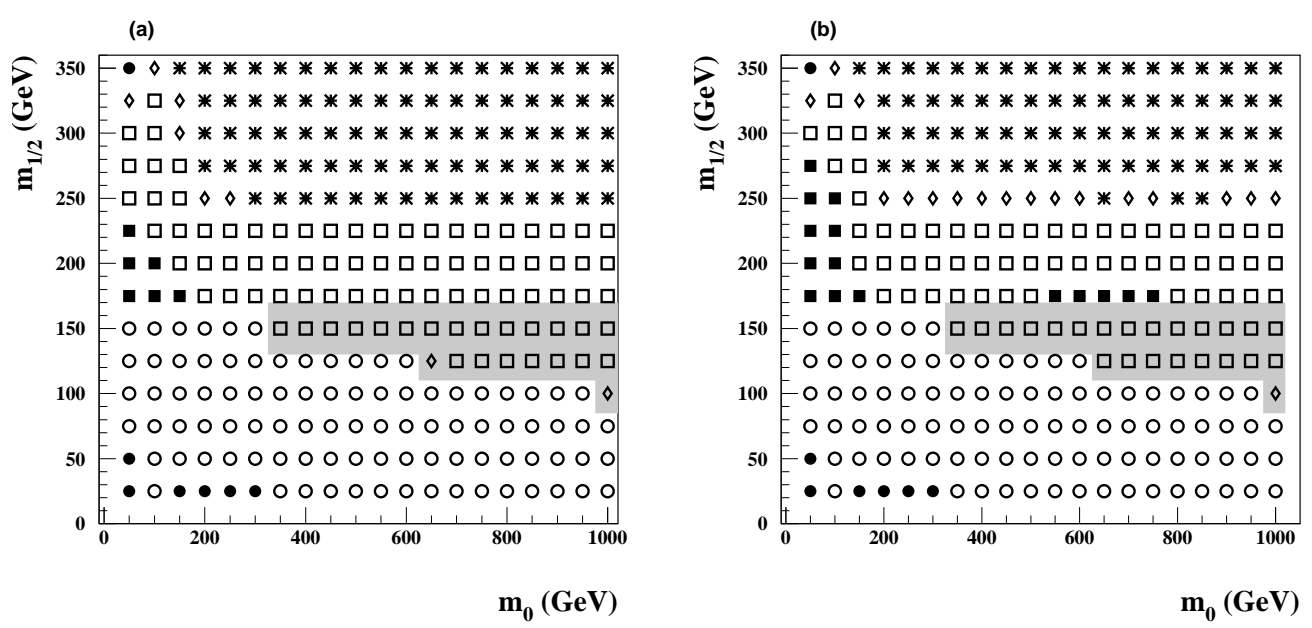

Figure 3: (a) Reach of Fermilab Tevatron Run II in the 4 or more lepton channel. (b) Combined trilepton and multilepton results. All parameters and conventions were chosen as in Fig. 2 .

drastic reduction of the $\tilde{\chi}_{2}^{0}$ branching ratio into leptons at large $m_{0}$ is compensated by the additional production of charged leptons in $\tilde{\chi}_{1}^{0}$ decays.

In Fig. 3(a) we present the Tevatron reach in the multilepton (four leptons or more) channel for the same parameters adopted in Fig. 2. As we can see, the multilepton reach is larger than the trilepton one, increasing the discovery potential at large values of $m_{1 / 2}$. 
For instance, the Tevatron reach at large $m_{0}$ is $\simeq 225 \mathrm{GeV}$ in our BRpV model while it is of the order of $150 \mathrm{GeV}$ in the MSSM. Moreover, unlike the trilepton signal, the discovery potential at small $m_{0}$ is also increased with respect to that of the MSSM. In this region it is clear that the reduction of the trilepton signal is largely due to the presence of additional isolated leptons. As in Fig. 2 the shaded area represent the region where displaced vertices could be used to further increase the sensitivity to BRpV. In Fig. B(b) we present the combined results for the trilepton and multilepton searches using the chi-square criteria. It is interesting to notice that the presence of $R$-parity violating interactions leads to a $5 \sigma$ SUSY discovery even at large $m_{0}$, a region where the usual $R$-parity conserving SUGRA model has no discovery potential at all. Notice the importance of combining trilepton and multilepton signals to achieve this conclusion.

\section{Conclusion}

We have analysed the production of multileptons ( $\geq 3 \ell$ with $\ell=e$ or $\mu$ ) in the simplest supergravity model with violation of $R$ parity at the Fermilab Tevatron. In this model, an effective bilinear term in the superpotential parameterises the explicit breaking of $R$ parity. Despite the small $R$-parity violating couplings needed to generate the neutrino masses indicated by current atmospheric neutrino data, the lightest supersymmetric particle is unstable and can decay inside the detector. This leads to a phenomenology quite distinct from that of the $R$-parity conserving scenario. We have quantified by how much the supersymmetric multilepton signals differ from the $R$-parity conserving expectations, displaying our results in the $m_{0} \otimes m_{1 / 2}$ plane. We have shown that the presence of bilinear $R$-parity violating interactions enhances the supersymmetric multilepton signals over most of the parameter space, specially at moderate and large $m_{0}$. These topologies are useful not only for discovery, but also to verify whether $R$ parity is conserved or not.

We have shown how the presence of $\mathrm{BRpV}$ interactions leads to a small suppression of the trilepton signal at small values of $m_{0}$ irrespective of the value of $\mathrm{BRpV}$ parameter $\epsilon_{\tau}$. This is due to the $\tilde{\chi}_{1}^{0}$ decay into $\nu b \bar{b}$. However, the $\tilde{\chi}_{1}^{0}$ decays lead to a drastically extended reach at large $m_{0}$ as a result of the LSP decay into $\tau u \bar{d}$. Moreover, the presence of additional isolated leptons in the signal allows us to look for multilepton events.

We have demonstrated that combining the trilepton and multilepton searches increases the Tevatron Run II sensitivity for most of SUGRA and $R$-parity breaking parameters. Note, however, that for neutrino masses in the range indicated by current atmospheric data one has a gap between the $\tilde{\chi}_{1}^{0}$ masses that can be probed at LEP2 (up to $40 \mathrm{GeV}$ or so) and those that can be studied at the Tevatron (above $70 \mathrm{GeV}$ or so): within this range the $\tilde{\chi}_{1}^{0}$ decay length is rather large, requiring the study of other topologies, like the presence of displaced vertices in the tracking system.

\section{Acknowledgments}

Research supported by Fundação de Amparo à Pesquisa do Estado de São Paulo (FAPESP), by Conselho Nacional de Desenvolvimento Científico e Tecnológico (CNPq), by Programa 
de Apoio a Núcleos de Excelência (PRONEX), by Spanish MCyT grant BFM2002-00345 and by the European Commission RTN network HPRN-CT-2000-00148. W.P. has been supported by the 'Erwin Schrödinger fellowship' No. J2272 of the 'Fonds zur Förderung der wissenschaftlichen Forschung' of Austria and partly by the Swiss 'Nationalfonds'. D.R. has been partially supported by UdeA-CODI Grant No. 381-8-10-02.

\section{References}

[1] C. S. Aulakh and R. N. Mohapatra, Phys. Lett. B 119, 13 (1982); L. J. Hall and M. Suzuki, Nucl. Phys. B 231, 41 (1984); G. G. Ross and J. W. Valle, Phys. Lett. B 151, 375 (1985); J. R. Ellis et al., Phys. Lett. B 150, 142 (1985).

[2] M. Hirsch et al., Phys. Rev. D 62, 113008 (2000) [Erratum-ibid. D 65, 119901 (2002)] [arXiv:hep-ph/0004115]; J. C. Romão et al., Phys. Rev. D 61, 071703 (2000) [arXiv:hep-ph/9907499]; M. A. Díaz et al., arXiv:hep-ph/0302021, Phys. Rev. D, in press

[3] A. Bartl et al., Nucl. Phys. B 600, 39 (2001) [arXiv:hep-ph/0007157], and references therein.

[4] W. Porod, M. Hirsch, J. Romão and J. W. Valle, Phys. Rev. D 63, 115004 (2001) [arXiv:hep-ph/0011248], and references therein.

[5] M. Hirsch, W. Porod, J. C. Romão and J. W. Valle, Phys. Rev. D 66 (2002) 095006 [arXiv:hep-ph/0207334].

[6] M. A. Díaz, J. C. Romão and J. W. Valle, Nucl. Phys. B 524, 23 (1998) [arXiv:hep-ph/9706315].

[7] H. Baer, M. Drees, F. Paige, P. Quintana and X. Tata, Phys. Rev. D 61, 095007 (2000) [arXiv:hep-ph/9906233].

[8] M. Maltoni, T. Schwetz, M. A. Tortola and J. W. Valle, Phys. Rev. D 67013011 (2003), [arXiv:hep-ph/0207227], and references therein.

[9] M. B. Magro, et al., JHEP 0309, 071 (2003) [arXiv:hep-ph/0304232].

[10] V. D. Barger, C. Kao and T.-J. Li, Phys. Lett. B433, 328 (1998) [arXiv:hep-ph/9804451];

V. D. Barger and C. Kao, Phys. Rev. D60, 115015 (1999) [arXiv:hep-ph/9811489].

[11] In the trilepton analysis, we reject events presenting more than three isolated charged leptons.

[12] T. Söstrand, Computer Phys. Commun. 82, 74 (1994).

[13] K. Hagiwara et al., Phys. Rev. D66, 010001 (2002). 